\title{
Systemic therapies for hepatocellular carcinoma: an evolving landscape
}

\author{
Filippo Pelizzaro', Giuliano Ramadori², Fabio Farinati ${ }^{1}$ \\ 'Department of Surgery, Oncology and Gastroenterology, Gastroenterology Unit, University of Padova, Padova 35128, Italy. \\ ${ }^{2}$ Department of Gastroenterology and Endocrinology, University Medical Center Goettingen, Goettingen D-37075, Germany.
}

Correspondence to: Prof. Fabio Farinati, Department of Surgery, Oncology and Gastroenterology, Gastroenterology Unit, University of Padova, Via Giustiniani 2, Padova 35128, Italy. E-mail: fabio.farinati@unipd.it

How to cite this article: Pelizzaro F, Ramadori G, Farinati F. Systemic therapies for hepatocellular carcinoma: an evolving landscape. Hepatoma Res 2021;7:36. https://dx.doi.org/10.20517/2394-5079.2021.24

Received: 22 Feb 2021 First Decision: 25 Apr 2021 Revised: 26 Apr 2021 Accepted: 26 Apr 2021 Published: 8 May 2021

Academic Editor: Guang-Wen Cao Copy Editor: Xi-Jun Chen Production Editor: Xi-Jun Chen

\begin{abstract}
In the last few years, there has been a significant widening of the landscape of systemic therapy for unresectable hepatocellular carcinoma (HCC) patients. After the landmark drug sorafenib, several other molecules have been approved for treatment in first-line (lenvatinib) and second-line (regorafenib, cabozantinib, and ramucirumab) regimens. Very recently, another important step forward has been made with the demonstration that the combination of an anti-programmed death ligand 1 and an anti-vascular endothelial growth factor (atezolizumab + bevacizumab) provides better survival results compared to sorafenib, thus becoming the new paradigm in first-line treatment of HCC. In consideration of this rapidly evolving situation, with the availability of many potential active drugs, the American Society of Clinical Oncology recently published a guideline in order to advise on the selection of systemic treatment options. However, also considering the uncertainties and the unmet needs in the current treatment of patients with advanced liver cancer is mandatory.
\end{abstract}

Keywords: Hepatocellular carcinoma, systemic therapies, tyrosine kinase inhibitors, immune checkpoint inhibitors, atezolizumab, bevacizumab, American Society of Clinical Oncology (ASCO)

The search for effective systemic therapy in hepatocellular carcinoma (HCC) patients has been very intense for many years, but all the initial drugs investigated (chemotherapy and hormonal compounds) proved to be ineffective $\mathrm{e}^{[1]}$. Among the most promising molecules, tyrosine kinase inhibitors (TKIs) attracted great attention due to their ability to block intracellular pathways involved in several biological activities in HCC 
cells ${ }^{[2]}$. In 2008 and 2009, for the first time, two randomized controlled trials (RCTs) showed that the TKI sorafenib was able to prolong overall survival (OS) compared to placebo in patients with $\mathrm{HCC}^{[3,4]}$. Following this milestone in the treatments of patients with HCC, several other molecules (brivanib, linifanib, sunitinib, and erlotinib) were compared against sorafenib in phase III trials, but failed to demonstrate a survival benefit, either due to lack of effectiveness or unacceptable toxicity ${ }^{[5]}$. Almost 10 years passed before additional advances in systemic treatment for HCC were achieved. In 2017-2019, four RCTs demonstrated positive results in first- and second-line setting with either TKIs (regorafenib, cabozantinib, and lenvatinib) or a VEGFR-2 antagonist (ramucirumab) ${ }^{[--9]}$. An additional step forward in the management of unresectable HCC patients was made last year with the positive result of the IMbrave150 trial, demonstrating a clear survival benefit of the combination of an anti-programmed death ligand 1 (PD-L1) drug (atezolizumab) and an anti-vascular endothelial growth factor (VEGF) molecule (bevacizumab), over sorafenib ${ }^{[10]}$.

The landscape of systemic therapies for HCC is rapidly evolving and the significant expansion of treatment possibilities in the last years make it difficult for clinicians to decide how to approach pharmacological treatment for a patient with HCC. With the aim to provide a guidance to the selection of first-line and subsequent second-line systemic therapies, the American Society of Clinical Oncology (ASCO) recently published its recommendations [Table 1] ${ }^{[11]}$.

Following the results of IMbrave150 trial, which demonstrated a dramatic improvement of OS in atezolizumab + bevacizumab arm compared to sorafenib $(\mathrm{HR}=0.58 ; 95 \% \mathrm{CI}: 0.42-0.79 ; P=0.0006)^{[10]}$, the combination therapy is recommended as the standard of care first-line treatment in patients with advanced HCC, Child-Pugh class A and Eastern Cooperative Oncology Group performance status (ECOG-PS) 0-1 ${ }^{[11]}$. The remarkably better OS, progression free survival (PFS), and response rate results obtained with the combination of atezolizumab + bevacizumab are groundbreaking, considering that previous trials with antiPD1 immune checkpoint inhibitorsICI alone (nivolumab and pembrolizumab) failed to show efficacy in first- and second-line ${ }^{[12,13]}$. In phase II trials, bevacizumab monotherapy showed promising response rates (about $14 \%$ ) in patients with advanced $\mathrm{HCC}^{[14-16]}$, thus confirming the central role of angiogenesis in the development and progression of liver cancer ${ }^{[17]}$. The rationale behind the combination of bevacizumab and ICI relies on the immunomodulatory effects of $\mathrm{VEGF}^{[18]}$. Beyond promoting neovascularization, VEGF produced in the tumor microenvironment is able to enhance the release of immunosuppressive cytokines and to reduce the proliferation and activation of $\mathrm{T}$ lymphocytes ${ }^{[19-21]}$. In addition, through its effect on angiogenesis, VEGF prevents the infiltration of antigen-activated $\mathrm{CD} 8+$ cells in the tumor tissue and creates a microenvironment that inhibits their function ${ }^{[21]}$. Therefore, the administration of VEGF inhibitors reprograms the immunosuppressive tumor microenvironment toward immunostimulation, and the administration of PD-1/PD-L1 antibodies under such conditions enhances antitumoral activity of T cells ${ }^{[18]}$. This may explain the high efficacy of combined therapy, which is also confirmed by the results of a phase Ib trial that showed significantly longer PFS in the atezolizumab and bevacizumab group than in patients receiving atezolizumab alon ${ }^{[22]}$. The superiority of atezolizumab + bevacizumab over other first-line treatment options (sorafenib, lenvatinib, and nivolumab), was recently confirmed in a systematic review and metanalysis ${ }^{[23]}$.

A major concern when using anti-VEGF drugs in cirrhotic patients is bleeding since upper gastrointestinal bleeding is a common and life-threatening complication in these patients. In IMbrave150 trial the incidence of upper gastrointestinal bleeding in atezolizumab + bevacizumab group was $7 \%$ compared to $4.5 \%$ in the sorafenib group, despite the exclusion from the study of patients with untreated or incompletely treated gastro-esophageal varices and high risk of bleeding ${ }^{[10]}$. Although similar rates of fatal treatment emergent adverse events were reported in the two arms, hemorrhage was more common in atezolizumab + 
Table 1. Summary of the ASCO guidelines recommendations ${ }^{[11]}$

\begin{tabular}{|c|c|c|c|}
\hline Recommendations & Type & $\begin{array}{l}\text { Evidence } \\
\text { quality }\end{array}$ & $\begin{array}{l}\text { Strength of } \\
\text { recommendation }\end{array}$ \\
\hline \multicolumn{4}{|l|}{ First-line therapy } \\
\hline $\begin{array}{l}\text { 1.1 Atezolizumab + bevacizumab as first-line therapy for most patients with } \\
\text { advanced HCC, Child-Pugh class A, ECOG-PS 0-1, following management of } \\
\text { esophageal varices when present }\end{array}$ & $\begin{array}{l}\text { Evidence-based, } \\
\text { benefits outweigh } \\
\text { harms }\end{array}$ & $\begin{array}{l}\text { Moderate to } \\
\text { high }\end{array}$ & Strong \\
\hline $\begin{array}{l}\text { 1.2 With contraindications to atezolizumab and/or bevacizumab, TKIs sorafenib } \\
\text { or lenvatinib as first-line therapy of patients with advanced HCC, Child-Pugh class } \\
\text { A, and ECOG-PS } 0-1\end{array}$ & $\begin{array}{l}\text { Evidence-based, } \\
\text { benefits outweigh } \\
\text { harms }\end{array}$ & Moderate & Strong \\
\hline \multicolumn{4}{|l|}{ Second-line therapy } \\
\hline $\begin{array}{l}\text { 2.1 Following first-line treatment with atezolizumab + bevacizumab, second-line } \\
\text { therapy with a TKI (sorafenib, lenvatinib, regorafenib, or cabozantinib) may be } \\
\text { recommended }\end{array}$ & $\begin{array}{l}\text { Informal consensus, } \\
\text { benefits outweigh } \\
\text { harms }\end{array}$ & Low & Weak \\
\hline $\begin{array}{l}\text { 2.2 Following first-line therapy with sorafenib or lenvatinib, second-line therapy } \\
\text { with another TKI (cabozantinib or regorafenib), ramucirumab (AFP } \geq 400 \\
\mathrm{ng} / \mathrm{mL} \text { ), or atezolizumab + bevacizumab may be recommended }\end{array}$ & $\begin{array}{l}\text { Informal consensus, } \\
\text { benefits outweigh } \\
\text { harms }\end{array}$ & $\begin{array}{l}\text { Low to } \\
\text { moderate }\end{array}$ & Weak \\
\hline $\begin{array}{l}\text { 2.3 Following first-line therapy with sorafenib or lenvatinib, pembrolizumab or } \\
\text { nivolumab are reasonable options that may be considered for appropriate } \\
\text { candidates }\end{array}$ & $\begin{array}{l}\text { Informal consensus, } \\
\text { benefits outweigh } \\
\text { harms }\end{array}$ & Low & Weak \\
\hline
\end{tabular}

HCC: Hepatocellular carcinoma; ECOG-PS: Eastern Cooperative Oncology Group performance status; TKIs: tyrosine kinase inhibitors; AFP: alphafetoprotein; ASCO: American Society of Clinical Oncology.

bevacizumab arm (four gastrointestinal and one subarachnoid) than in sorafenib arm (one peritoneal ${ }^{[24]}$. For these reasons, a careful assessment of gastro-esophageal varices, and their management when present, should be performed before starting treatment with anti-VEGF drugs.

In patients who cannot be treated with atezolizumab + bevacizumab due to contraindications, ASCO recommend the use of sorafenib or lenvatinib ${ }^{[11]}$. A not negligible proportion of patients with unresectable HCC falls in this category, considered the very restrictive criteria for treatment with ICI in clinical trials. An analysis of the Italian Liver Cancer (ITA.LI.CA) database showed that, in a first-line scenario, patients amenable to treatment with ICI in the real-life clinical practice, adopting the same inclusion/exclusion criteria of RCTs, are no more than one-third of potential candidates ${ }^{[25,26]}$. Therefore, the role of TKIs in the treatment of HCC patients seems far from sunset, with sorafenib and lenvatinib being still a valuable option in first-line treatment of Child-Pugh class A and ECOG-PS 0-1 patients. In the non-inferiority REFLECT trial ${ }^{[8]}$, no differences in OS between lenvatinib and sorafenib were demonstrated (HR $=0.92$; 95\% CI: 0.791.06). Nevertheless, some considerations about the results of this trial may help in the selection between the two drugs: in lenvatinib group PFS was significantly longer ( $\mathrm{HR}=0.64$; 95\%CI: 0.55-0.76), objective response rate was significantly higher $(\mathrm{OR}=5.01 ; 95 \% \mathrm{CI}$ : 3.59-7.01), and despite the fact that lenvatinib treated patients were more likely to discontinue treatment due to adverse events, their median duration of treatment was longer (5.7 months $v s .3 .7$ months ${ }^{[8]}$. On the contrary, the inclusion criteria of the SHARP trial ${ }^{[3]}$ were less restrictive, in particular with respect to patients with ECOG-PS 2, > 50\% of liver occupation, main portal vein invasion, and clear bile duct invasion. Moreover, when choosing therapy it should be considered that randomized controlled data on second-line treatment exists exclusively for patients who received sorafenib in first-line ${ }^{[6,7,9]}$. Only a post-hoc analysis of the REFLECT trial data has been published, showing that sorafenib may be a valuable option after lenvatinib, despite the very low number of patients analyzed $^{[27]}$. Beyond all, the choice between sorafenib and lenvatinib should be made balancing safety issues (since the two treatments have different risk of specific adverse events) and expected treatment benefits, through a discussion involving the physician and patient. 
Regorafenib $^{[6]}$, cabozantinib ${ }^{[7]}$, and ramucirumab ${ }^{[9]}$ are all second-line treatments approved for patients previously treated with sorafenib, which was the standard of care at the time RCTs were conducted. Nowadays, the changed paradigm of first-line treatment after IMbrave150 trial makes all the recommendation for second-line therapies characterized by low quality of evidence and weak strength, being essentially Expert Panel opinions ${ }^{[11]}$. A second-line therapy with a TKI after atezolizumab + bevacizumab will probably work, in consideration of its different mechanism of action, but there is uncertainty regarding the best molecule to administer (i.e., sorafenib, lenvatinib, cabozantinib, or ramucirumab). Guidelines state that, after atezolizumab + bevacizumab, sorafenib or lenvatinib may be offered, but also regorafenib or cabozantinib are reasonable options ${ }^{[1]}$. After a first line with sorafenib or lenvatinib, a second-line with another TKI (regorafenib or cabozantinib) or ramucirumab (in patients with AFP $\geq 400 \mathrm{ng} / \mathrm{mL}$ ) may be prescribed, with atezolizumab + bevacizumab as an alternative option ${ }^{[11]}$. This latter recommendation derives from the fact that it is likely that most patients considered for atezolizumab + bevacizumab in the second-line setting did not have access to this combination when they started firstline treatment. However, it should be again considered that there are no studies evaluating the therapeutic sequence, except if sorafenib is used as first-line. Moreover, the Expert Panel suggests to consider pembrolizumab or nivolumab following first-line with sorafenib or lenvatinib (in appropriate candidates who have contraindications to or cannot tolerate TKIs) ${ }^{[11]}$. The FDA granted accelerated approval to pembrolizumab after a first-line treatment with sorafenib following a phase II trial (Keynote 224) demonstrating encouraging tumor response rate and durability of response ${ }^{[28]}$. However, in the subsequent phase III trial (Keynote 240), although confirming a high response rate (18\%), pembrolizumab failed to demonstrate an advantage in OS and PFS compared to placebo as per prespecified statistical plan ${ }^{[13]}$. In a second-line setting, nivolumab was approved by FDA following a phase I/IIb dose escalation and expansion trial (Checkmate 040) demonstrating relevant response rates ${ }^{[29]}$, but no phase III trial data are currently available. In the Checkmate 459 trial $^{[12]}$, comparing nivolumab and sorafenib in first-line, despite the high median OS in nivolumab treated patients (16.4 months), the statistically significant superiority over sorafenib was not achieved $(\mathrm{HR}=0.85 ; 95 \% \mathrm{CI}$ : 0.72-1.02; $P=0.0752)$. Although one can agree on the potential utility of pembrolizumab and nivolumab in the treatment of patients who have contraindications to or cannot tolerate TKIs, currently there are no randomized data demonstrating a survival advantage for these drugs. Therefore, the interpretation of these indications should be very cautious. In third-line, the only available option is cabozantinib since in the CELESTIAL trial patients previously treated with one or two other systemic therapy regimens were enrolled ${ }^{[7]}$. No specific recommendation on third-line therapy has been included in ASCO guidelines, but for the acknowledgement that it may be considered in healthy patients with preserved liver function after multidisciplinary decision ${ }^{[11]}$.

Despite all these recent relevant advances, a wide area of uncertainty and unmet needs still exists, especially regarding the substantial lack of data to support treatment benefits in Child-Pugh class B patients. The only successful registration trial which was not limited to patients with compensated liver disease was the SHARP trial, but only $5 \%$ of sorafenib treated patients was Child-Pugh class $\mathrm{B}^{[3]}$. In real-life clinical scenarios, sorafenib is frequently prescribed regardless of liver function and safety seems not to be different in mildly decompensated liver disease ${ }^{[3,31]}$, although these patients demonstrated worse survival ${ }^{[32]}$. For other drugs, very limited data in Child-Pugh class B patients are available ${ }^{[33-36]}$. In addition, high quality evidence in the systemic treatment of HCC in patients with different comorbidities excluded from trials (i.e., solid organ transplantation, HIV, autoimmune diseases, cardiovascular diseases, bleeding history, and hemodialysis) is missing ${ }^{[37]}$. In patients with recurrence of HCC after liver transplantation, retrospective studies suggests that sorafenib and regorafenib are safe and moderately effective in this setting ${ }^{[38,39]}$. On the other hand, the use of ICI after liver transplantation may be problematic, since the activation of the immune system may lead to organ rejection, as demonstrated by the available case series ${ }^{[40]}$. Therefore, TKIs probably represent the therapy of choice in this category of patients. In the future, the identification of reliable 
biomarkers of treatment response would be very useful in guiding treatment decisions. Lastly, another point that has to be considered is the cost-effectiveness of subsequent different lines of treatment, which still remains to be carefully determined.

Significant advances in the treatments of unresectable HCC have been made in the last few years and additional, even greater, changes in this landscape are on the way. After the sorafenib era that lasted more than 10 years, the atezolizumab + bevacizumab combination therapy has emerged as the new paradigm in the first-line systemic treatment. Although at present this therapy is becoming the standard of care, some uncertainties are still present such as the patients' amenability to this treatment in real-life clinical scenarios, the best second-line approach, or the specific treatment of HCC developing after HCV eradication with direct acting antivirals. We are witnessing a sort of rapidly evolving revolution in the field of systemic treatment for HCC, but many other research efforts are still to be made in order to ensure an ever-better survival and quality of life for patients with advanced liver cancer.

\section{DECLARATIONS}

\section{Authors' contributions}

Made substantial contributions to conception and design of the study and performed data analysis and interpretation: Pelizzaro F, Ramadori G, Farinati F

All the authors equally contributed to this work.

\section{Availability of data and materials}

Not applicable.

\section{Financial support and sponsorship}

None.

\section{Conflicts of interest}

All authors declared that there are no conflicts of interest.

\section{Ethical approval and consent to participate}

Not applicable.

\section{Consent for publication}

Not applicable.

\section{Copyright}

(c) The Author(s) 2021.

\section{REFERENCES}

1. Association For The Study Of The Liver, European Organisation For Research And Treatment Of Cancer. EASL-EORTC clinical practice guidelines: management of hepatocellular carcinoma. J Hepatol 2012;56:908-43. DOI PubMed

2. Ramadori G, Füzesi L, Grabbe E, Pieler T, Armbrust T. Successful treatment of hepatocellular carcinoma with the tyrosine kinase inhibitor imatinib in a patient with liver cirrhosis. Anticancer Drugs 2004;15:405-9. DOI PubMed

3. Llovet JM, Ricci S, Mazzaferro V, et al. Sorafenib in advanced hepatocellular carcinoma. N Engl J Med 2008;359:378-90. DOI PubMed

4. Cheng AL, Kang YK, Chen Z, et al. Efficacy and safety of sorafenib in patients in the Asia-Pacific region with advanced hepatocellular carcinoma: a phase III randomised, double-blind, placebo-controlled trial. Lancet Oncol 2009;10:25-34. DOI PubMed

5. Villanueva A. Hepatocellular carcinoma. N Engl J Med 2019;380:1450-62. DOI PubMed

6. Bruix J, Qin S, Merle P, et al; RESORCE Investigators. Regorafenib for patients with hepatocellular carcinoma who progressed on sorafenib treatment (RESORCE): a randomised, double-blind, placebo-controlled, phase 3 trial. Lancet 2017;389:56-66. DOI PubMed

7. Abou-Alfa GK, Meyer T, Cheng A-L, et al. Cabozantinib in patients with advanced and progressing hepatocellular carcinoma. $N$ Engl 
J Med 2018;379:54-63. DOI PubMed PMC

8. Kudo M, Finn RS, Qin S, et al. Lenvatinib versus sorafenib in first-line treatment of patients with unresectable hepatocellular carcinoma: a randomised phase 3 non-inferiority trial. Lancet 2018;391:1163-73. DOI PubMed

9. Zhu AX, Kang YK, Yen CJ, et al. Ramucirumab after sorafenib in patients with advanced hepatocellular carcinoma and increased $\alpha$ fetoprotein concentrations ( REACH-2 ): a randomised, double-blind, placebo-controlled, phase 3 trial. Lancet Oncol 2019;20:282-96. DOI PubMed

10. Finn RS, Qin S, Ikeda M, et al. Atezolizumab plus bevacizumab in unresectable hepatocellular carcinoma. $N$ Engl J Med 2020;382:1894-905. DOI PubMed

11. Gordan JD, Kennedy EB, Abou-Alfa GK, et al. Systemic therapy for advanced hepatocellula carcinoma: ASCO Guideline. J Clin Oncol 2020;38:4317-45. DOI PubMed

12. Yau T, Park JW, Finn RS, et al. LBA38_PR - CheckMate 459: a randomized, multi-center phase 3 study of nivolumab (NIVO) vs sorafenib (SOR) as first-line (1L) treatment in patients (pts) with advanced hepatocellular carcinoma (aHCC). Ann Oncol 2019;30 (suppl_5):v874-5. DOI

13. Finn RS, Ryoo BY, Merle P, et al; KEYNOTE-240 investigators. Pembrolizumab as second-line therapy in patients with advanced hepatocellular carcinoma in KEYNOTE-240: a randomized, double-blind, phase III trial. J Clin Oncol 2020;38:193-202. DOI PubMed

14. Siegel AB, Cohen EI, Ocean A, et al. Phase II trial evaluating the clinical and biologic effects of bevacizumab in unresectable hepatocellular carcinoma. J Clin Oncol 2008;26:2992-8. DOI PubMed PMC

15. Boige V, Malka D, Bourredjem A, et al. Efficacy, safety, and biomarkers of single - agent bevacizumab therapy in patients with advanced hepatocellular carcinoma. Oncologist 2012;17:1063-72. DOI PubMed PMC

16. Finn RS, Zhu AX. Targeting angiogenesis in hepatocellular carcinoma: focus on VEGF and bevacizumab. Expert Rev Anticancer Ther 2009;9:503-9. DOI PubMed

17. Zhu AX, Duda DG, Sahani DV, Jain RK. HCC and angiogenesis: possible targets and future directions. Nat Rev Clin Oncol 2011;8:292-301. DOI PubMed PMC

18. Kudo M. Scientific rationale for combined immunotherapy with PD-1/PD-L1 antibodies and vegf inhibitors in advanced hepatocellular carcinoma. Cancers (Basel) 2020;12:1089. DOI PubMed PMC

19. Fukumura D, Kloepper J, Amoozgar Z, Duda DG, Jain RK. Enhancing cancer immunotherapy using antiangiogenics: Opportunities and challenges. Nat Rev Clin Oncol 2018;15:325-40. DOI PubMed PMC

20. Chouaib S, Messai Y, Couve S, Escudier B, Hasmim M, Noman MZ. Hypoxia promotes tumor growth in linking angiogenesis to immune escape. Front Immunol 2012;3:21. DOI PubMed PMC

21. Voron T, Marcheteau E, Pernot S, et al. Control of the immune response by pro-angiogenic factors. Front Oncol 2014;4:70. DOI PubMed PMC

22. Lee MS, Ryoo BY, Hsu CH, et al; GO30140 investigators. Atezolizumab with or without bevacizumab in unresectable hepatocellular carcinoma (GO30140): an open-label, multicentre, phase 1b study. Lancet Oncol 2020;21:808-20. DOI PubMed

23. Sonbol MB, Riaz I Bin, Naqvi SAA, et al. Systemic therapy and sequencing options in advanced hepatocellular carcinoma: a systematic review and network meta-analysis. JAMA Oncol 2020;6:e204930. DOI PubMed PMC

24. Casak SJ, Donoghue M, Fashoyin-Aje L, et al. FDA approval summary: atezolizumab plus bevacizumab for the treatment of patients with advanced unresectable or metastatic hepatocellular carcinoma. Clin Cancer Res 2020;27:1836-41. DOI PubMed

25. Giannini EG, Aglitti A, Borzio M, et al; Associazione Italiana per lo Studio del Fegato (AISF) HCC Special Interest Group. Overview of immune checkpoint inhibitors therapy for hepatocellular carcinoma, and the ITA.LI.ca cohort derived estimate of amenability rate to immune checkpoint inhibitors in clinical practice. Cancers (Basel) 2019;11:1689. DOI PubMed PMC

26. Tovoli F, De Lorenzo S, Trevisani F. Immunotherapy with checkpoint inhibitors for hepatocellular carcinoma: where are we now? Vaccines (Basel) 2020;8:578. DOI PubMed PMC

27. Alsina A, Kudo M, Vogel A, et al. Effects of subsequent systemic anticancer medication following first-line lenvatinib: a post hoc responder analysis from the phase 3 REFLECT study in unresectable hepatocellular carcinoma. Liver Cancer 2020;9:93-104. DOI PubMed PMC

28. Zhu AX, Finn RS, Edeline J, et al; KEYNOTE-224 investigators. Pembrolizumab in patients with advanced hepatocellular carcinoma previously treated with sorafenib (KEYNOTE-224): a non-randomised, open-label phase 2 trial. Lancet Oncol 2018;19:940-52. DOI PubMed

29. El-Khoueiry AB, Sangro B, Yau T, et al. Nivolumab in patients with advanced hepatocellular carcinoma (CheckMate 040): an openlabel, non-comparative, phase 1/2 dose escalation and expansion trial. Lancet 2017;389:2492-502. DOI PubMed PMC

30. Marrero JA, Kudo M, Venook AP, et al. Observational registry of sorafenib use in clinical practice across Child-Pugh subgroups: The GIDEON study. J Hepatol 2016;65:1140-7. DOI PubMed

31. Labeur TA, Achterbergh R, Takkenberg B, Van Delden O, Mathôt R, Klümpen HJ. Sorafenib for patients with hepatocellular carcinoma and Child-Pugh B liver cirrhosis: lessons learned from a terminated study. Oncologist 2020;25:e1274-9. DOI PubMed $\mathrm{PMC}$

32. McNamara MG, Slagter AE, Nuttall C, et al. Sorafenib as first-line therapy in patients with advanced Child-Pugh B hepatocellular carcinoma-a meta-analysis. Eur J Cancer 2018;105:1-9. DOI PubMed

33. Chen SC, Chao Y, Chen MH. Lenvatinib for the treatment of HCC: A single institute experience. J Clin Oncol 2019;37:e15611. DOI

34. Zhu AX, Baron AD, Malfertheiner P, et al. Ramucirumab as second-line treatment in patients with advanced hepatocellular carcinoma analysis of REACH trial results by child-pugh score. JAMA Oncol 2017;3:235-43. DOI PubMed 
35. El-Khoueiry A, Meyer T, Cheng A, et al. Outcomes for patients with advanced hepatocellular carcinoma and Child-Pugh B liver function in the phase 3 CELESTIAL study of cabozantinib vs placebo. Abstr ESMO 2020;31:S220. DOI

36. Kudo M, Matilla A, Santoro A, et al. Checkmate-040: Nivolumab (NIVO) in patients (pts) with advanced hepatocellular carcinoma (aHCC) and Child-Pugh B (CPB) status. J Clin Oncol 2019;37:327. DOI

37. Rimassa L, Personeni N, Czauderna C, Foerster F, Galle P. Systemic treatment of HCC in special populations. J Hepatol 2020;74:93143. DOI PubMed

38. Iavarone M, Invernizzi F, Czauderna C, et al. Preliminary experience on safety of regorafenib after sorafenib failure in recurrent hepatocellular carcinoma after liver transplantation. Am J Transplant 2019;19:3176-84. DOI PubMed

39. Sposito C, Mariani L, Germini A, et al. Comparative efficacy of sorafenib versus best supportive care in recurrent hepatocellular carcinoma after liver transplantation: a case-control study. J Hepatol 2013;59:59-66. DOI PubMed

40. Pinter M, Scheiner B, Peck-Radosavljevic M. Immunotherapy for advanced hepatocellular carcinoma: a focus on special subgroups. Gut 2021;70:204-14. DOI PubMed PMC 